\title{
An Optical Coherence Tomography and Fundus Autofluorescence Imaging Study of Peripapillary Acute Zonal Occult Outer Retinopathy
}

\author{
Shinji Makino Yoshiaki Tanaka Hironobu Tampo \\ Department of Ophthalmology, Jichi Medical University, Tochigi, Japan
}

\section{Key Words}

Acute zonal occult outer retinopathy · Fundus autofluorescence imaging · Fluorescein angiography · Indocyanine green angiography · Optical coherence tomography · Visual field . Multifocal electroretinogram

\begin{abstract}
Purpose: To evaluate peripapillary acute zonal occult outer retinopathy in a 67-year-old man. Methods: Images were obtained using fundus photography, fluorescein angiography (FA) and indocyanine green angiography (ICGA), fundus autofluorescence (FAF) imaging, and optical coherence tomography (OCT). Visual field testing and multifocal electroretinogram (mfERG) were also performed to evaluate retinal function. Results: Fundus photographs showed subtle pigmentary changes in the peripapillary region, while FAF imaging showed clearly defined hypofluorescent areas in the peripapillary region. Intense hyperfluorescent lesions were also seen underneath hypofluorescent areas. A transmission defect with a granular hyperfluorescence was visible on FA, and ICGA showed hypofluorescence within the lesion. The outer border of the peripapillary zone appeared to block the underlying choroidal fluorescence. The photoreceptor inner segment/outer segment line was absent on OCT images from this area in both eyes, and hyperreflective punctate drusen-like materials were present at the outer border of the peripapillary zone. Visual field testing by Goldmann perimetry showed blind-spot enlargement, and mfERG showed corresponding amplitude reductions. Conclusions: We speculate that the intense hyperautofluorescent material at the outer border of the peripapillary zone might be accumulated drusenoid lipofuscin.
\end{abstract}


Makino et al:: An Optical Coherence Tomography and Fundus Autofluorescence Imaging Study of Peripapillary Acute Zonal Occult Outer Retinopathy

\section{Introduction}

Acute zonal occult outer retinopathy (AZOOR) is an ocular syndrome characterized by an acute decrease in outer retinal function in one or more retinal zones. Initially, minimal or no fundus changes occur, but enlarged blind spots, abnormalities on electroretinogram (ERG), and permanent visual field loss often occur in this slowly progressing form of retinal pigment epithelium (RPE) degeneration [1, 2]. Optical coherence tomography (OCT) images from eyes with AZOOR show a loss or irregularity of the photoreceptor inner segment/outer segment (IS/OS) line in areas corresponding to reduced multifocal ERG (mfERG) responses and visual field defects [3-7]. Visible fundus changes, including vessel narrowing, perivascular sheathing, and mottled pigmentation, may follow in later stages of the disease [2]. Most patients affected by AZOOR are young females [1].

Spaide et al. [8] described a prominent accumulation of material at the outer border of the peripapillary zone that was hypofluorescent on fluorescein angiography (FA) and consistent, both in color and autofluorescent characteristics, with lipofuscin. To the best of our knowledge, no cases of a prominent drusen-like material accumulation at the outer border of the peripapillary zone, as examined by OCT, have been reported in the literature. Here, we report OCT findings, in combination with other standard ophthalmic examinations, in a patient with AZOOR.

\section{Case Report}

A 67-year-old man presented with a several-month history of an expanding visual field defect in both eyes. His personal and family history and systemic evaluation were unremarkable. His visual acuity was 0.7 in both eyes and intraocular pressure measurements were normal. Slit-lamp examination showed cortical opacities in both lenses, and fundus photographs showed subtle pigmentary alterations in the peripapillary regions (fig. $1 \mathrm{a}, \mathrm{m}$ ). Additional affected zones were also found near the superotemporal vascular arcade in the left eye. Both fundus autofluorescence (FAF; fig. 1c, o) and infrared FAF (IR-FAF; fig. 1b, n) imaging showed clearly defined hypofluorescent areas in the peripapillary regions. Hyperfluorescent lesions at the outer border of hypofluorescent areas were also visible on both FAF and IR-FAF imaging, and mottled hyperfluorescent areas were detected in the region on FAF imaging. FA showed a transmission defect with a granular hyperfluorescence (fig. 1d, e, p, q), and the outer border of the peripapillary zone was not clearly shown due to a blocking phenomenon. Indocyanine green angiography (ICGA) showed hypofluorescence within the lesion, but no reduction in fluorescence of the underlying choroidal vessels (fig. $1 \mathrm{f}, \mathrm{g}, \mathrm{r}, \mathrm{s})$. The outer border of the peripapillary zone appeared to block the underlying choroidal fluorescence. The Heidelberg Retina Angiograph 2 (Heidelberg Engineering, Heidelberg, Germany) was used to perform IR-FAF, FAF, FA, and ICGA imaging.

OCT (RS-3000; Nidek, Japan) was also performed and demonstrated that the IS/OS line was absent in the areas of IR-FAF and FAF hypofluorescence in both eyes (fig. $1 \mathrm{~h}, \mathrm{i}, \mathrm{t}, \mathrm{u}$ ). Both vertical and horizontal OCT scans showed the presence of hyperreflective punctate drusenlike material at the outer border of the peripapillary zone (fig. 1t, u, fig. 2a, b). Visual field testing by Goldmann perimetry showed blind-spot enlargement in both eyes (fig. 1j, v) and a central relative scotoma in the right eye. The size of the visual field defect corresponded to the size of the fundus lesions, as seen on autofluorescent, ICGA, and FA imaging. Reduced amplitude was also noted in mfERG (LE-4100; Tomey, Japan) for areas corresponding to visual field defects (fig. 1k, l, w, x). Six months later, affected peripapillary regions in the left 
Makino et al.: An Optical Coherence Tomography and Fundus Autofluorescence Imaging Study of Peripapillary Acute Zonal Occult Outer Retinopathy

eye had extended through the macula and had become confluent with the initially separate lesion near the superotemporal vascular arcade.

\section{Discussion}

An elderly man had findings consistent with AZOOR. Spaide et al. [8] previously described an AZOOR patient with prominent accumulation of material at the outer border of the peripapillary zone. The material was hypofluorescent on FA images and was consistent, both in color and autofluorescence, with lipofuscin. Cohen et al. [9] described a peripapillary AZOOR and choroidal neovascularization. Their fundus changes $[8,9]$ were very similar to the case presented here, but, in our case, fundus photograph did not clearly show yellowish drusen-like material at the outer border of the lesion. However, the outer border of the peripapillary zone was clearly defined on IR-FAF, FAF, FA, and ICGA images. To the best of our knowledge, the literature does not contain descriptions of cases with prominent accumulation of drusen-like material at the peripapillary zone outer border, as imaged by OCT. On OCT, hyperreflective punctate drusen-like material was present at the level of the RPE in this case. In FAF images, hyperfluorescence was presumed to result from accumulated lipofuscin in RPE cells and to represent metabolic activity of RPE related to photoreceptor outer segment turnover. Conversely, hypofluorescence is thought to represent areas of decreased RPE metabolism resulting from photoreceptor and/or RPE atrophy. Therefore, RPE autofluorescence has been used as a marker for RPE/photoreceptor complex integrity. Abnormal FAF findings in our patient were suggestive of photoreceptor structural integrity loss and included IS/OS junction and photoreceptor outer segment abnormalities. Spaide et al. [8] suggested that AZOOR causes the formation of an area of lipofuscin-laden cells, and subsequent RPE cell death, at the border of the expanding lesion. Our OCT findings support this theory, but it is not clear whether the hyperreflective punctate drusen-like material represents acute or advanced changes resulting from AZOOR. Further studies in additional cases are necessary to answer this question.

The majority of patients who develop AZOOR are women [1]. Out of 130 published cases in which sex was specified, 99 (76\%) involved women and 31 (24\%) involved men. Additionally, most AZOOR patients are young adults, with an average age of 36.7 years (range 13-79 years) in the 103 published cases. Therefore, we initially suspected that our 67-year-old patient was in the late stages of AZOOR. However, this may not have been the case as our patient showed enlargement and confluence of affected areas over a 6-month period.

Fujiwara et al. [10] investigated how AZOOR is represented on FAF and OCT in 19 eyes. Abnormalities on FAF imaging were detected in 17 (89\%) of 19 eyes and they fell into the following three broad categories: only peripapillary involvement ( $\mathrm{n}=9$ eyes, 53\%), peripheral involvement $(\mathrm{n}=7$ eyes, $41 \%)$, and posterior pole involvement not primarily centered on the optic nerve ( $\mathrm{n}=3$ eyes, 18\%). Of the eyes with FAF abnormalities, 14 eyes $(82 \%)$ had broad areas of hypoautofluorescence and 3 eyes (18\%) had coarse granular regions of mixed hyper- and hypoautofluorescence. The boundary of autofluorescent abnormalities was well-defined in 11 eyes (65\%), with 6 (35\%) of these having a hyperautofluorescent border. Nine eyes (53\%) showed progression of hypoautofluorescent areas during follow-up. In our case, hypofluorescent peripapillary areas had a mottled pattern of hyperautofluorescence, and intense hyperautofluorescent lesions were detected at the outer border of hypofluorescent areas. 
Makino et al.: An Optical Coherence Tomography and Fundus Autofluorescence Imaging Study of Peripapillary Acute Zonal Occult Outer Retinopathy

Fujiwara et al. [10] also described IS/OS boundary abnormalities in all 19 eyes studied. Fourteen eyes (74\%) showed some absence of the IS/OS boundary and 5 eyes (26\%) showed attenuation of the IS/OS boundary on OCT scans. Globular hyperreflective aggregates were found in the inner nuclear layer of 9 eyes (47\%) and between the ganglion cell and inner plexiform layers in 7 eyes (37\%). Longer hyporeflective bands within the ganglion cell layer were also observed in 10 eyes (53\%). In general, these abnormalities occurred over areas where severe attenuation or loss of the photoreceptor layer had occurred. In all 12 eyes with an abnormal inner retinal structure, the outer nuclear layer was either absent or attenuated. In our case, globular hyperreflective aggregates were not detected in the inner nuclear layer, but intensely hyperautofluorescent material at the level of the RPE was present. In conclusion, we speculate that intensely hyperautofluorescent material at the outer border of the peripapillary zone might be drusenoid lipofuscin accumulation.

\section{Disclosure Statement}

The authors have no conflicts of interest to disclose.

\section{References}

1 Monson DM, Smith JR: Acute zonal occult outer retinopathy. Surv Ophthalmol 2011;56:23-35.

2 Gass JD, Agarwal A, Scott IU: Acute zonal occult outer retinopathy: a long-term follow-up study. Am J Ophthalmol 2002;134:329-339.

-3 Li D, Kishi S: Loss of photoreceptor outer segment in acute zonal occult outer retinopathy. Arch Ophthalmol 2007;125:1194-1200.

4 Spaide RF, Koizumi H, Freund KB: Photoreceptor outer segment abnormalities as a cause of blind spot enlargement in acute zonal occult outer retinopathy-complex diseases. Am J Ophthalmol 2008;146:111120.

5 Takai Y, Ishiko S, Kagokawa H, Fukui K, Takahashi A, Yoshida A: Morphological study of acute zonal occult outer retinopathy (AZOOR) by multiplanar optical coherence tomography. Acta Ophthalmol 2009;87:408418.

6 Ohta K, Sato A, Fukui E: Spectral domain optical coherence tomographic findings at convalescent stage of acute zonal occult outer retinopathy. Clin Ophthalmol 2009;3:423-428.

-7 So K, Shinoda K, Matsumoto CS, Satofuka S, Imamura Y, Mizota A: Focal functional and microstructural changes of photoreceptors in eyes with acute zonal occult outer retinopathy. Case Rep Ophthalmol 2011;2:307-313.

-8 Spaide RF: Collateral damage in acute zonal occult outer retinopathy. Am J Ophthalmol 2004;138:887-889.

$\checkmark 9$ Cohen SY, Jampol LM: Choroidal neovascularization in peripapillary acute zonal occult outer retinopathy. Retinal Cases and Brief Reports 2007;220-222.

10 Fujiwara T, Imamura Y, Giovinazzo VJ, Spaide RF: Fundus autofluorescence and optical coherence tomographic findings in acute zonal occult outer retinopathy. Retina 2010;30:1206-1216. 


\section{Case Reports in \\ Ophthalmology}

\begin{tabular}{l|l}
\hline \multicolumn{2}{l}{ Case Rep Ophthalmol 2013;4:11-16 } \\
\hline DOI: $\underline{10.1159 / 000346731}$ & $\begin{array}{l}\text { ○ 2013 S. Karger AG, Basel } \\
\text { www.karger.com/cop }\end{array}$ \\
\hline
\end{tabular}

Makino et al:: An Optical Coherence Tomography and Fundus Autofluorescence Imaging Study of Peripapillary Acute Zonal Occult Outer Retinopathy

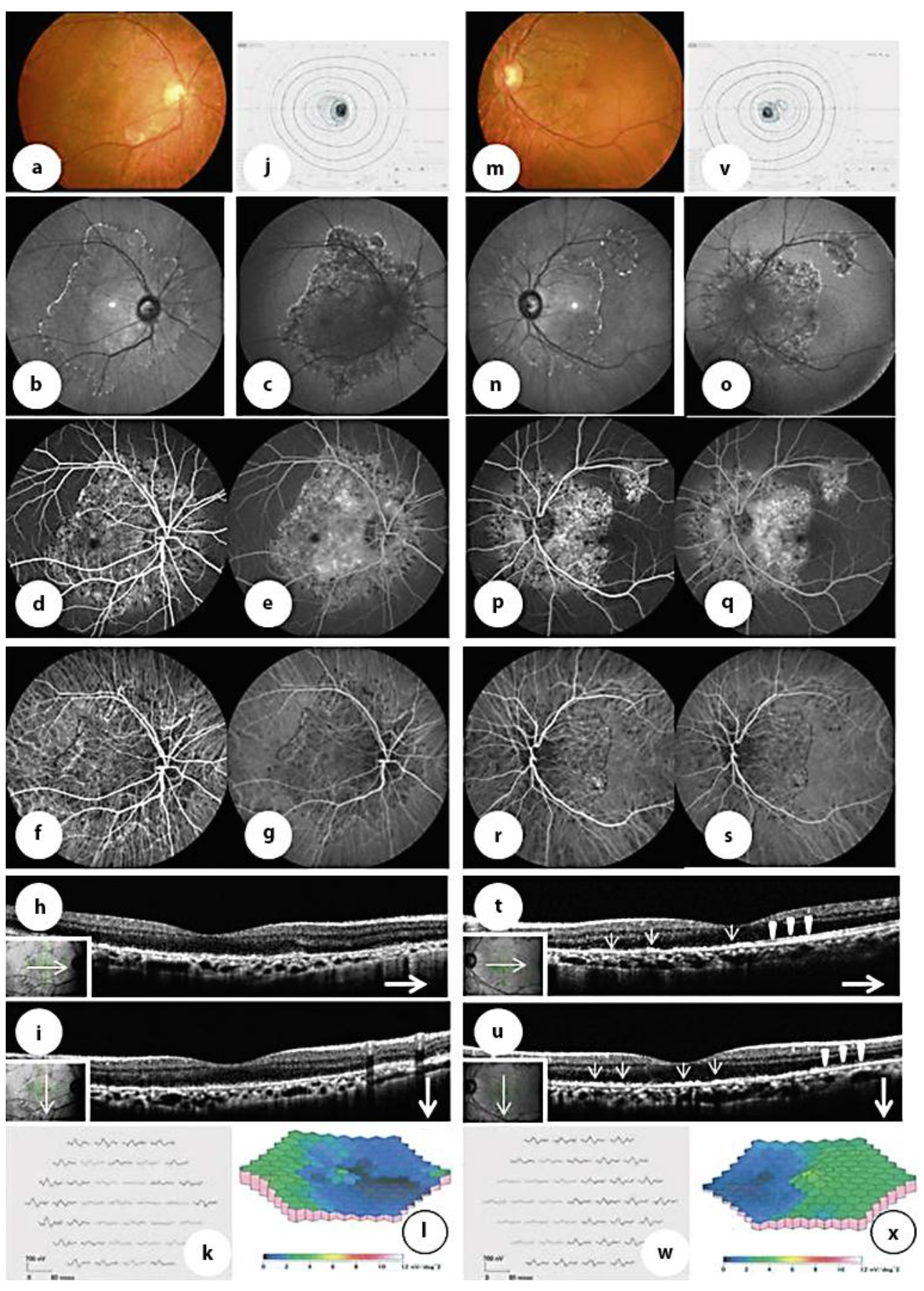

Fig. 1. Findings in a 67-year-old man with peripapillary AZOOR in the right (a-I) and left ( $\mathbf{m}-\mathbf{x})$ eye. a, $\mathbf{m}$ Fundus photographs show subtle pigmentary alterations in the peripapillary region. $\mathbf{b}, \mathbf{n}$ Infra-red FAF (IR-FAF) imaging shows clearly defined hypofluorescent areas in the peripapillary regions. Hyperautofluorescent lesions can also be seen at the outer border of hypofluorescent areas. c, o FAF imaging shows clearly defined hypofluorescent areas in the peripapillary regions and intense hyperautofluorescent lesions at the outer border of hypofluorescent areas. FA early- (d, p) and late-phase (e, q) images show a transmission defect with granular hyperfluorescence. ICGA early- $(\mathbf{f}, \mathbf{r})$ and late-phase $(\mathbf{g}, \mathbf{s})$ images show hypofluorescence within the lesion and no dimming of the underlying larger choroidal vessels' fluorescence. The outer border of the peripapillary zone appears to block the underlying choroidal fluorescence. OCT horizontal $(\mathbf{h}, \mathbf{t})$ and vertical $(\mathbf{i}, \mathbf{u})$ scans show an absent photoreceptor IS/OS line 
Makino et al:: An Optical Coherence Tomography and Fundus Autofluorescence Imaging Study of Peripapillary Acute Zonal Occult Outer Retinopathy

within AZOOR lesions. Arrowheads indicate an intact photoreceptor IS/OS line in an area of intact retina in the left eye. Hyperreflective punctate drusen-like material was present (arrows). j, v Goldmann perimetry showed blind-spot enlargement in both eyes and a central relative scotoma in the right eye. mfERG response arrays (k, w) and 3-dimensional plots (I, $\mathbf{x}$ ) showed reduced responses in areas corresponding to visual field defects.
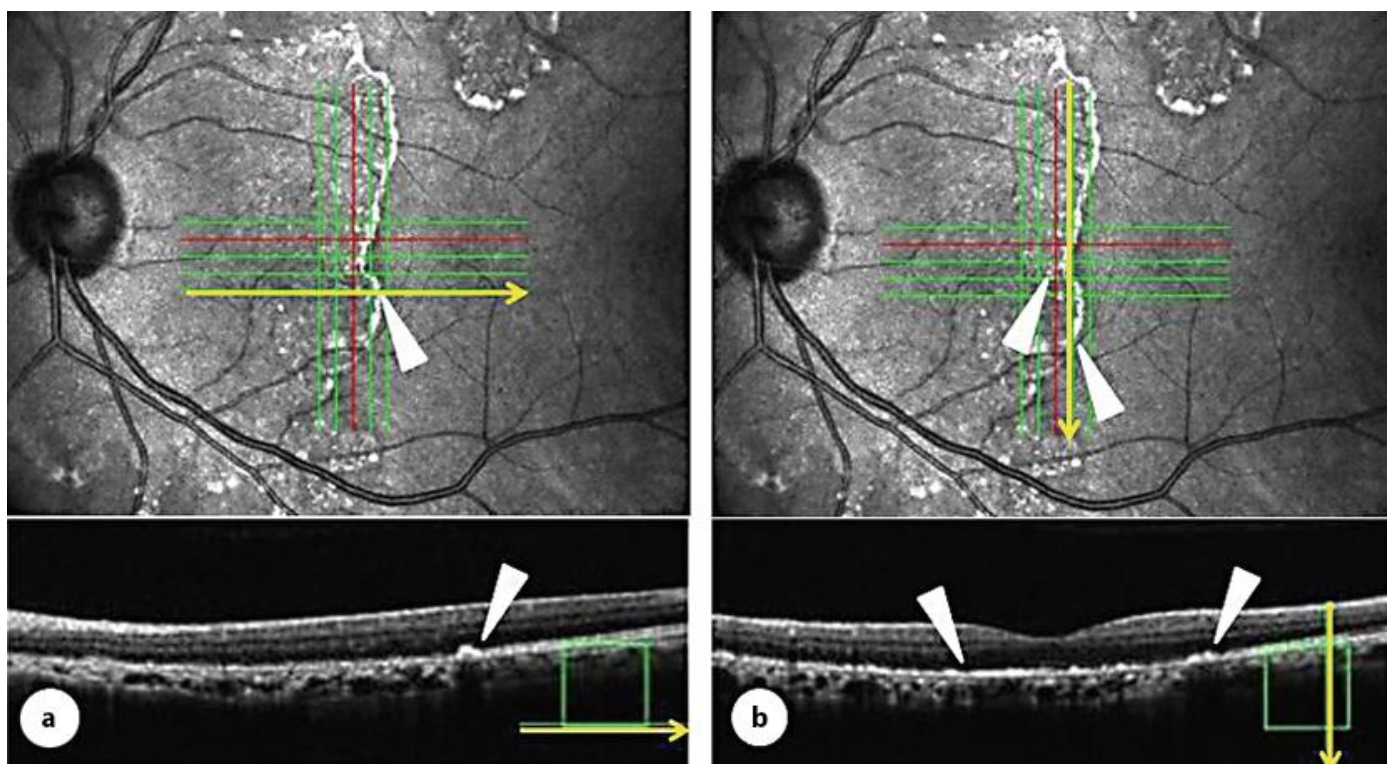

Fig. 2. High magnification of OCT horizontal (a) and vertical (b) scans showing the presence of hyperreflective punctate drusen-like materials (arrowheads). 